\title{
ICEBERG DETECTION WITH L-BAND ALOS-2 DATA USING THE DUAL-POL RATIO ANOMALY DETECTOR.
}

\author{
Armando Marino \\ The University of Stirling, Natural Sciences, Stirling, United Kingdom
}

\begin{abstract}
Icebergs represent a danger to navigation in cold waters. Detection and tracking of large icebergs using space-borne scatterometers, altimeters and synthetic aperture radar (SAR) systems have seen a large amount of work in the last decades. However, the identification of small icebergs is still challenging especially when these are embedded in sea ice. In this work, a recently proposed iceberg detector the intensity Dual-Pol Ratio Anomaly Detector (iDPolRAD) is tested using ALOS-2 L-band data. The detector is designed for dual-polarized incoherent SAR images, however in this work we want to investigate the loss of performance with respect to using coherent data. Therefore different versions of the iDPolRAD requiring quad-pol data are proposed here and tested.

The ALOS-2 data were acquired on the East Coast of Greenland, where a large number of icebergs are visible in the images. The results show that different polarisations could bring different information and therefore the availability of dual-pol could improve the detection although a quad-pol algorithm will not be operational at the moment due to the limited size of the swath.
\end{abstract}

Index Terms - SAR, polarimetry, iceberg detection, ALOS-2

\section{SAR POLARIMETRY}

Based on its polarization characteristics, a single target can be characterized using the scattering (Sinclair) matrix or equivalently a scattering vector $\underline{k}$ [1]. In the lexicographic basis system and in case of reciprocity and monostatic system, the scattering vector is $\underline{k}_{L}=[H H, \sqrt{2} H V, V V]$, where $H$ stands for linear horizontal and $V$ for linear vertical (therefore the $H V$ image is obtained transmitting a linear vertical polarization and receiving the linear horizontal one). $H H$ and $V V$ are often referred to as co-channels and $H V$ or $V H$ are the cross-channels.

The data were provided by JAXA under the project number 1151 . ALOS-2 Product - (c)JAXA 2017, all rights reserved.

(C) 2018 IEEE. Personal use of this material is permitted. Permission from IEEE must be obtained for all other uses, in any current or future media, including reprinting/ republishing this material for advertising or promotional purposes, creating new collective works, for resale or redistribution to servers or lists, or reuse of any copyrighted component of this work in other works.

\section{DUAL-POLARIZATION RATIO ANOMALY DETECTOR (DPOLRAD)}

\subsection{Dimensionless detector}

An algorithm was proposed for the detection of small icebergs in sea ice. The intensity Dual-Polarization Ratio Anomaly Detector (iDPolRAD) was already successfully used with Sentinel-1 data to detect icebergs embedded in sea ice [2]. This work presents the very first test of the iDPolRAD with L-band data. Additionally, we developed different versions based on the availability of different polarimetric channels.

The algorithm is based on the observation that icebergs exhibit a different polarimetric behaviour compared to the sea ice [3]. Specifically, the cross polarization channel and the ratio between cross- and co-polarizations (here referred to as depolarization ratio) increases. Icebergs are composed by a volume of fresh water and the presence of reflective planes (mostly wet surfaces) with random orientations. they are therefore expected to have a polarimetric bahcscattering that is different from the one of the sea or sea ice surface scattering $[4,5]$. Since there is volume scattering and the orientations of the corners are generally random, icebergs are expected to have a large cross-polarisation contribution [3].

Two boxcar filters are applied over the HV and HH intensity images, exploiting two different window sizes: a smaller window or test window $w_{\text {test }}$ and a larger window or training window $w_{\text {train }}$. The detector can be written as:

$$
\Lambda=\frac{\left\langle|H V|^{2}\right\rangle_{\text {test }}-\left\langle|H V|^{2}\right\rangle_{\text {train }}}{\left\langle|H H|^{2}\right\rangle_{\text {train }}}>T_{\Lambda} .
$$

where \langle\rangle$_{\text {test }}$ and \langle\rangle$_{\text {train }}$ identify the spatial average using the test and training windows respectively and $T_{\Lambda}$ is a threshold.

After some mathematical manipulations it is possible to gain some physical understanding of the proposed formula. If the averages are expressed explicitly the following equation can be derived:

$$
\Lambda=\rho_{\text {ring }} \frac{1+c}{R \rho^{-1}+c R H V^{-1}}-\rho_{\text {train }}
$$

$\rho$ stands for cross-over-co polarization ratio, in the following defined as depolarization ratio. As mentioned previously, 
this observable is sensitive to the presence of orientated structures. $R \rho$ is the ratio between the $\rho$ inside the test area over the one in the ring around the test area (i.e. $R \rho=\frac{\rho_{\text {test }}}{\rho_{\text {ring }}}$ ). $R H V$ is the ratio of the $\mathrm{HV}$ intensity in the test area over the ring area (i.e. $R H V=\frac{\left\langle|H V|^{2}\right\rangle_{\text {test }}}{\left\langle|H V|^{2}\right\rangle_{\text {ring }}}$ ). $c$ is a factor such that $N_{\text {train }}=c N_{\text {test }}$ where $N_{\text {train }}$ and $N_{\text {test }}$ are the number of pixels inside the training and test windows. $\rho_{\text {ring }}$ and $\rho_{\text {train }}$ are the depolarization ratios in the ring and the entire training windows respectively.

In [2] is demonstrated that if an iceberg enters the test window, the value of $\Lambda$ increases triggering a detection. Please note that this detector does not need the phase information of each polarimetric channels but only their magnitude. It is therefore appropriate for systems that do not acquire the polarisation channels coherently.

\subsection{Contrast enhancement: HV-DPolRAD}

$\Lambda$ is large when there is an increase in volume or oriented scattering, equals to zero on homogeneous targets and is negative if there is a decrease in oriented scattering. Such detector is built as a ratio between intensities and therefore it is scale invariant. This is a very valuable property for a polarimetric observable, however scale invariance may be disadvantageous for some detection task. For instance, if the signal is very low and close to the noise floor, the detector may become noisy. An easy way out is by multiplying the detector by an intensity or magnitude image. In this context, the cross polarization channel should be preferred because it shows a higher contrast between icebergs and clutter:

$$
I=\Lambda \cdot\left\langle|H V|^{2}\right\rangle
$$

In this version, if a pixel of the HV intensity image presents an anomaly in volume or oriented reflections, then it is multiplied by a large number. If it presents a homogeneous area, then it is multiplied by zero and if it presents a decrease in volume or oriented reflections, then it becomes negative. This enhances the contrast between anomalies in volume/orientedreflections and clutter.

\subsection{Using different polarisation channels}

One of the aim of this work is to explore if the detection performance improves when different polarimetric channels are used. In particular, we are interested in evaluating the results of the combination of the co-polarisation channels $\mathrm{HH}$ and VV. To do this we need quad-polarimetric data that we acquired using ALOS-2 JAXA data. Please note that at he moment there is no satellite system that can acquire the copolarised channels with very large swaths and therefore the following analysis may only have a limited operational applicability to cover few strategic areas. Nevertheless we are interested in analysing this results to better understand the reduction of detection performance when using $\mathrm{HH}$ and $\mathrm{HV}$.
If only $\mathrm{HH}$ and VV are available we could design a detector that is focused on separating surface to horizontal dihedral scattering. The latter is expected from icebergs due to the horizontal double bounce with the sea (or sea ice) surface. However if the angle between the lower side of the iceberg vertical wall and the line of sight is not 90 degrees, the produced scattering may have an HV component much higher than the HH-VV component. It is interesting therefore to understand if $\mathrm{HV}$ or $\mathrm{HH}-\mathrm{VV}$ are best to detect the iceberg-sea double bounce scattering.

The detector can be easily adapted as

$$
\Lambda=\frac{\left\langle|H H-V V|^{2}\right\rangle_{\text {test }}-\left\langle|H H-V V|^{2}\right\rangle_{\text {train }}}{\left\langle|H H+V V|^{2}\right\rangle_{\text {train }}}>T_{\Lambda}
$$

The same derivation can be carried out leading to the equation

$$
\Lambda=\beta_{\text {ring }} \frac{1+c}{R \beta^{-1}+c R D^{-1}}-\beta_{\text {train }}
$$

$\beta$ stands for dihedral-over-surface polarization ratio. $R \beta$ is the ratio between the $\beta$ inside the test area over the one in the ring around the test area (i.e. $R \beta=\frac{\beta_{\text {test }}}{\beta_{\text {ring }}}$ ). $R D$ is the ratio of the $\mathrm{HH}-\mathrm{VV}$ intensity in the test area over the ring area (i.e. $R D=\frac{\left\langle|H H-V V|^{2}\right\rangle_{\text {test }}}{\left\langle|H H-V V|^{2}\right\rangle_{\text {ring }}}$ ). $c$ is a factor such that $N_{\text {train }}=c N_{\text {test }}$ where $N_{\text {train }}$ and $N_{\text {test }}$ are the number of pixels inside the training and test windows. $\beta_{\text {ring }}$ and $\beta_{\text {train }}$ are the depolarization ratios in the ring and the entire training windows respectively. We call this iDPolRAD2 and all the physical interpretations done for the iDPolRAD could be repeated here, substituting the phrase "volume and oriented reflections" with "horizontal reflections".

\section{TEST WITH ALOS-2 DATA}

\subsection{Presentation of data}

In this section, the algorithm is tested using subregions of quad-polarized ALOS-2 images. The resolution is approximately $8 m \times 5 m$. 10 images of icebergs were acquired under the 2013 ALOS- 2 call of opportunity. The location of the data is in Eastern Greenland near the basin of the Kangerlussuaq glacier. This is one of the most productive glaciers and several icebergs get grounded near the shore. Monitoring grounded icebergs is important to forecast the number of icebergs when they get released in spring. For the sake of brevity only two acquisitions will be presented here.

\subsection{Results}

In this preliminary study, only two dates from the $12^{\text {th }}$ of June 2015 and the $3^{\text {rd }}$ of August 2015 are analysed. Icebergs were visually identified using the high resolution SAR data. 
Among other things we looked at features as rims in the nearer range and shadows in the farther range.

Figure 1 presents the magnitude of $\mathrm{HH}$ and $\mathrm{HV}$ for the $12^{\text {th }}$ of June acquisition. The image shows the Greenland coast on the right hand side and sea covered by sea ice.

At the bottom left of Figure 1 we can observe how the iDPolRAD enhances the contrast between icebergs and sea ice. The test window considers $7 \times 7$ pixels, while the training window is $55 \times 55$ pixels. The scaling used for this image is exactly the same exploited for the HV magnitude. The image appear darker, because the sea ice clutter is strongly reduced. In this images, when the iDPolRAD is negative (i.e. reduction of volume or oriented reflections) the iDPolRAD is set to zero. On the other hand, bright isolated points remain bright. It is interesting to see how some of the icebergs embedded in sea ice and barely visible in the HV image become much easier to detect in the iDPolRAD image.

The same analysis is repeated for the data on the $3^{\text {rd }}$ of August and presented in Figure 2. It is interesting to see how the three icebergs inside the very bright background (middle of the image) can be easily visible after the filtering.

\subsection{Different polarisation channels}

The final test is dedicated to the detector using the combination of $\mathrm{HH}$ and VV, the iDPolRAD2. The results for the $12^{\text {th }}$ of June and $3^{r d}$ of August datesets are presented at the bottom right of Figure 1 and Figure 2 respectively.

It is interesting to observe that the filtering results are slightly different. There are some icebergs that are better detected by the original iDPolRAD and some that are more visible in iDPolRAD2. This complementarity suggests that in an ideal situation we may want to acquire quad-pol and use both detectors. However, at the moment the availability of large swaths constrain to the use of dual-pol incoherent images, i.e. iDPolRAD.

\section{CONCLUSIONS}

In this work we have tested a recently proposed iceberg detector iDPolRAD with L-band data. Additionally, we have evaluate different versions of the iDPolRAD which makes use of different polarisation channels. To perform this analysis we have used ALOS-2 data acquired on the East Coats of Greenland where it is possible to observe several icebergs.

The original detector seems to be applicable to L-band data. Additionally the evaluation of different polarisation channels allows to see that a richer information could be retrieved if quad-pol data were available. Preliminary results seems to show that the loss of performance will not reduce drastically the iceberg detection performance. However, further analysis will need to be done in order to quantify the amount of performance loss when dual-pol incoherent data are exploited.

\section{ACKNOWLEDGEMENTS}

The data were provided by the project number 1151. ALOS-2 Product - C)JAXA 2017, all rights reserved.

\section{REFERENCES}

[1] S. R. Cloude, Polarisation: Applications in Remote Sensing, Oxford University Press, Oxford, UK, 2009.

[2] A. Marino, W Dierking, and C. Wesche, "A depolarization ratio anomaly detector to identify icebergs in sea ice using dual-polarization SAR images," vol. 54, no. 9, pp. 5602-5615, 2016.

[3] W. Dierking and C. Wesche, "C-Band radar polarimetry useful for detection of icebergs in sea ice?," IEEE Transactions and Geoscience and Remote Sensing, vol. 52, no. 1, pp. 25-37, Jan 2014.

[4] F. Nunziata, M. Migliaccio, and C.E. Brown, "Reflection symmetry for polarimetric observation of man-made metallic targets at sea," IEEE Journal of Oceanic Engineering, vol. 37, no. 3, pp. 384-394, July 2012.

[5] A. Marino, "A notch filter for ship detection with polarimetric SAR data," IEEE Journal of Selected Topics in Applied Earth Observations and Remote Sensing, vol. 6, no. 3, pp. 1219 - 1232, June 2013. 

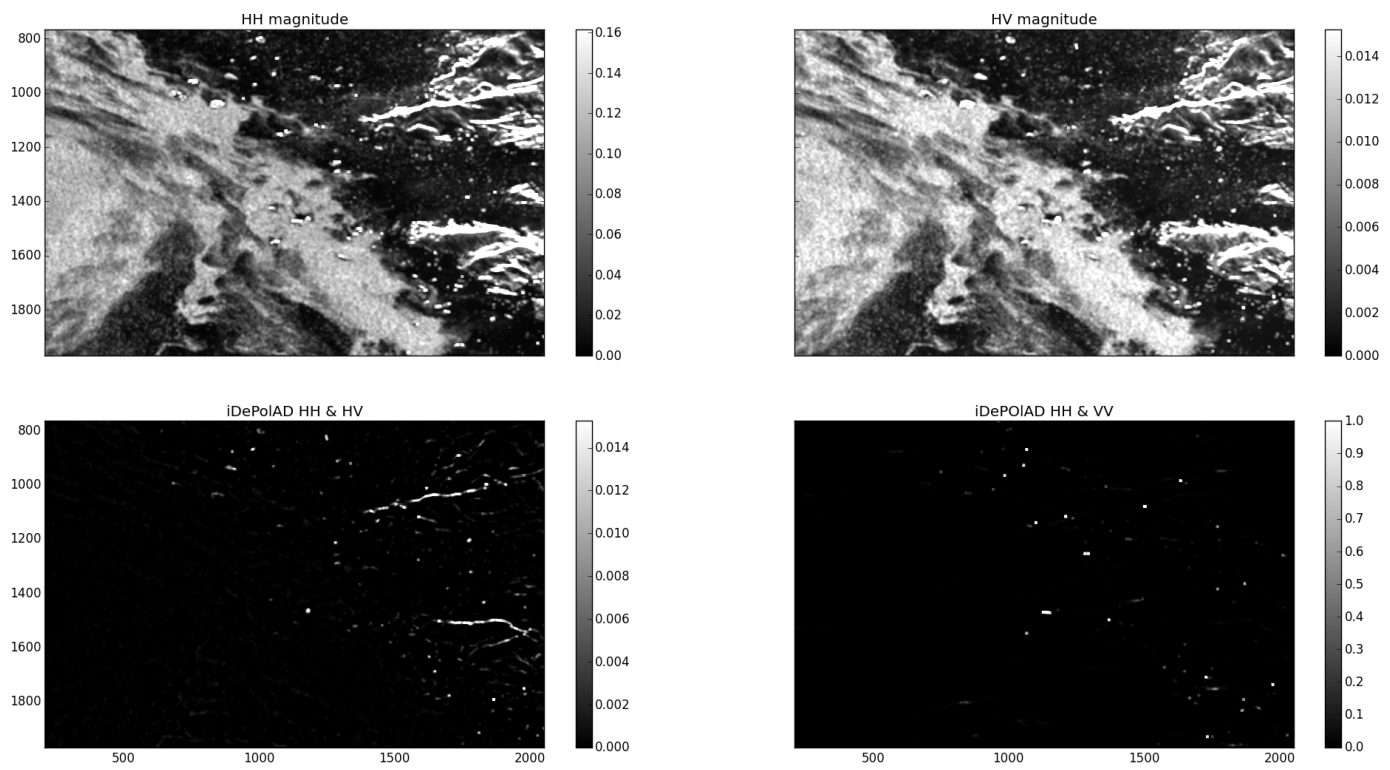

Fig. 1. Detection results with iDPolRAD using ALOS-2 quad-pol images(Eastern Greenland, 12/06/2015). (Top left) HH magnitude; (Top right) HV magnitude; (Bottom left) iDPolRAD, using the HH and HV polarisation channels; (Bottom right) iDPolRAD, using the HH and VV polarisation channels. Boxcar filter: $7 \times 7$ pixels. ALOS-2 Product - (c)
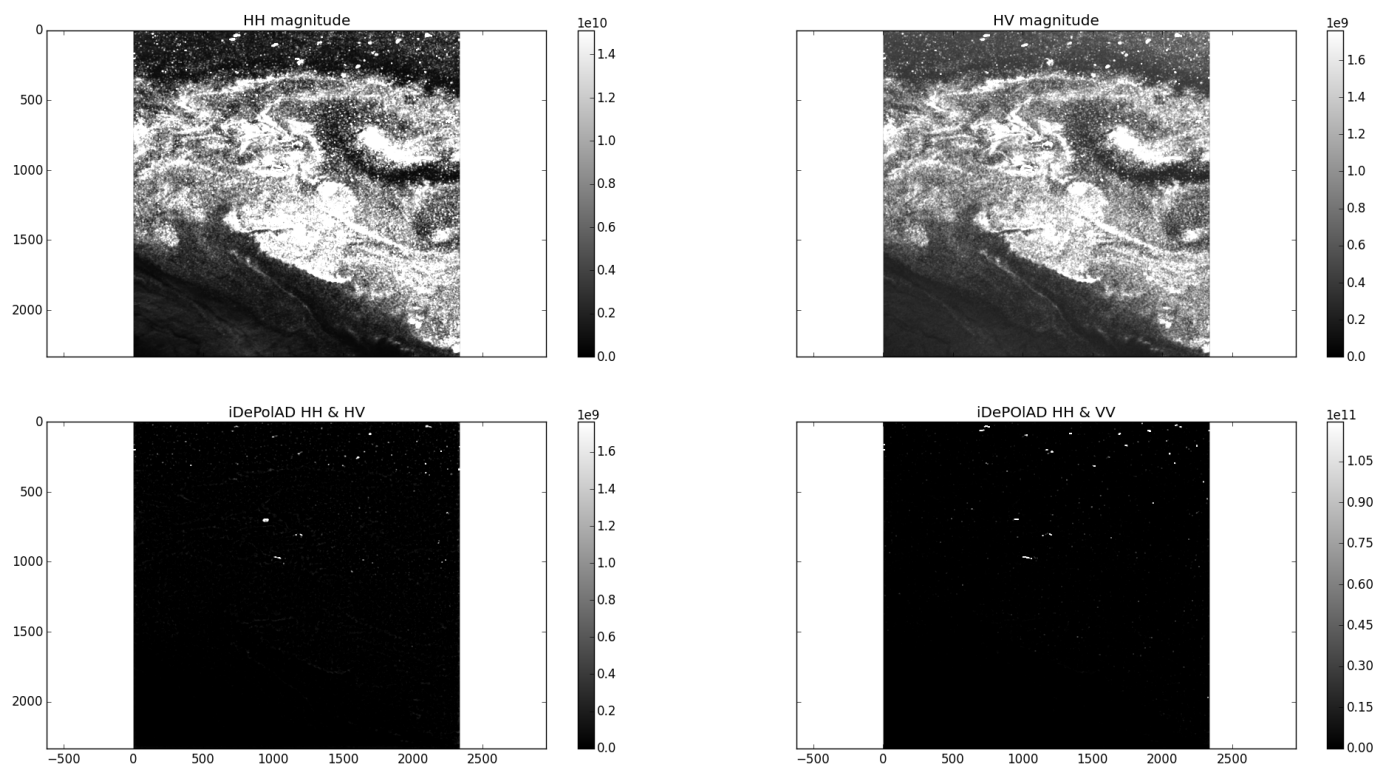

Fig. 2. Detection results with iDPolRAD using ALOS-2 quad-pol images(Eastern Greenland, 03/08/2015). (Top left) HH magnitude; (Top right) HV magnitude; (Bottom left) iDPolRAD, using the HH and HV polarisation channels; (Bottom right) iDPolRAD, using the HH and VV polarisation channels. Boxcar filter: $7 \times 7$ pixels. ALOS-2 Product - (C) 